\title{
A PROPERTY OF WEAK SOLUTIONS FOR SOME PARABOLIC EQUATIONS OF HIGHER ORDER
}

\author{
LU-SAN CHEN
}

To Professor Kiyoshi Noshiro on the occasion of his 60th birthday

1. Recently Aronson [1] proved the uniqueness property of weak solutions of the initial boundary value problem for second order parabolic equations with discontinuous coefficients. An analogous result to Aronson's was proved by Kuroda [4] in the case of some parabolic equations of higher order, where the method due to Aronson [2] plays an essential role. In this paper, under the same idea we shall be concerned with the asymptotic behavior of weak solutions for parabolic equations of higher order of the divergence form, when the data are prescribed on a portion of a time-like surface.

2. We use the symbol $x$ to denote a point $\left(x_{1}, \ldots, x_{n}\right)$ of the $n$-dimensional Euclidean space $E^{n}$, and $t$ to denote a point on the real line $(-\infty, \infty)$. Let $\mathscr{D}\left(\subset E^{n}\right)$ be a bounded domain and let $\Omega$ be the cylindrical domain $\mathscr{D} \times[0, \infty)$ in the $(n+1)$-dimensional Euclidean space $E^{n} \times(-\infty, \infty)$.

Consider a parabolic differential equation

$$
L u \equiv \frac{\partial u}{\partial t}-\sum_{\substack{|\alpha| \leqslant s \\|\beta| \leqslant s}} D_{x}^{\alpha}\left(a_{\alpha, \beta} D_{x}^{\beta} u\right)=f
$$

of order $2 s$ in $\Omega$, where $\alpha=\left(\alpha_{1}, \ldots, \alpha_{n}\right)$ is a multi-index of non-negative integers, $|\alpha|=\alpha_{1}+\cdots+\alpha_{n}$, and

$$
D_{x}^{\alpha}=\frac{\partial^{|\alpha|}}{\partial x_{1}^{\alpha_{1}} \cdots \partial x_{n}^{\alpha_{n}}}
$$

We shall assume that all the coefficients $a_{\alpha \beta}=a_{\alpha \beta}(x, t)$ are bounded measurable functions in $\Omega$ and that there exists a some positive constant $c$ such that

$$
\sum_{|\alpha|=|\beta|=s} a_{\alpha \beta} \xi_{1}^{\alpha_{1}+\beta_{1}} \cdots \xi_{n}^{\alpha_{n}+\beta_{n}} \geqq c\left(\xi_{1}^{2}+\cdots+\xi^{2}\right)^{s}
$$

for any real vector $\xi=\left(\xi_{1}, \ldots, \xi_{n}\right)$ and at every point in $\Omega$. Let $f$ be a func-

Received March 2, 1966. 
tion of $x, t$ and the derivatives $D_{x}^{\gamma} u(|\gamma| \leqq s)$ of the unknown function $u$ such that, for $u \in L^{2}\left[0, \infty ; H_{0}^{s, 2}(\mathscr{D})\right] \cap L^{\infty}\left[0, \infty ; L^{2}(\mathscr{D})\right]$, the function $f$ belongs to $L^{2}(\Omega)$ as a function in $\Omega$. We refer definition of these function spaces to $[2],[4]$.

If $u \in L^{2}\left[0, \infty ; H_{0}^{s, 2}(\mathscr{D})\right] \cap L^{\infty}\left[0, \infty ; L^{2}(\mathscr{D})\right]$ satisfies

$$
\begin{aligned}
& \int_{0}^{T^{\prime \prime}} \int_{g}\left(-u \frac{\partial \varphi}{\partial t}-\sum_{\substack{|\alpha| \leq s \\
|<| \leqslant s}}(-1)^{|\alpha|} a_{\alpha \beta} D_{x}^{\beta} u D_{x}^{\alpha} \varphi\right) d x d t \\
= & \int_{g} u(x, 0) \varphi(x, 0) d x-\int_{g} u\left(x, T^{\prime \prime}\right) \varphi\left(x, T^{\prime \prime}\right) d x+\int_{0}^{T^{\prime \prime}} \int_{g} f \varphi d x d t
\end{aligned}
$$

for any $T^{\prime \prime}(>0)$ and for any $\varphi \in H^{1,2}\left[0, \infty: H_{0}^{s, 2}(\mathscr{D})\right]$, then the function $u$ is said to be a weak solution of the equation (1) in $\Omega$ with boundary value zero.

Now we can prove the following theorem.

TheOREM. Let all the coefficients $a_{\alpha \beta}$ in (1) be continuous in the closure $\bar{Q}$ of $\Omega$ and assume that, in $\Omega$

$$
f^{2} \leqq k \sum_{|\alpha| \leqq_{\delta}}\left|D_{x}^{\alpha} u\right|^{2}
$$

for some positive constant $k$. If $s$ is even and if the weak solution $u$ of $(1)$ in $\Omega$ with boundary value zero and satisfies

$$
\lim _{T \rightarrow \infty} \int_{2} e^{2 \lambda T} u^{2}(x, T) d x=0
$$

for any $\lambda>0$, then $u$ is identically equal to zero in $\Omega$.

3. Proof of Theorem. We put

$$
u^{*}(x, t)=\left\{\begin{array}{c}
u(x, t), \text { if } t \in\left[0, T^{\prime \prime}\right] \\
0, \text { if } t \notin\left[0, T^{\prime \prime}\right] .
\end{array}\right.
$$

Let $j_{\varepsilon}(t)$ be an infinitely many times differentiable even function of a real variable $t$ with support $|t| \leqq \varepsilon$. In addition, we assume that $\int_{-\varepsilon}^{\varepsilon} j_{\varepsilon}(t)=1$. We put $\varphi(x, t)=e^{\lambda t} \int_{-\infty}^{\infty} e^{\lambda \tau} j_{\varepsilon}(t-\tau) u^{*}(x, \tau) d \tau=e^{\lambda t} \int_{0}^{T^{\prime \prime}} e^{\lambda \tau} j_{\varepsilon}(t-\tau) u(x, \tau) d \tau$ for a $\lambda>0$. It is not difficult to see that $\varphi \in H^{1,2}\left[0, \infty ; H_{0}^{s, 2}(\mathscr{D})\right]$. Hence the equality (3) is valid for the function $\varphi$ thus defined.

On the first term of the left hand side in (3), we get 


$$
\begin{gathered}
\int_{0}^{T^{\prime \prime}} \int_{g}-u \frac{\partial \varphi}{\partial t} d x d t=\int_{0}^{T^{\prime \prime}} \int_{2} \int_{0}^{T^{\prime \prime}}-e^{\lambda t} u(x, t) j_{\varepsilon}^{\prime}(t-\tau) e^{\lambda \tau} u(x, \tau) d \tau d x d t \\
-\lambda \int_{0}^{T^{\prime \prime \prime}} \int_{2} e^{2 \lambda t} u^{2} d x d t .
\end{gathered}
$$

From the fact that $j_{\varepsilon}(t)$ is even, we see that $j_{\varepsilon}^{\prime}(t)$ is odd. Hence it is easy to see that the first term in the right hand side of the above vanishes. Therefore we get

$$
\lim _{\varepsilon \rightarrow 0} \int_{0}^{\eta^{\prime \prime}} \int_{\mathscr{2}}-u \frac{\partial \varphi}{\partial t} d x d t=-\lambda \int_{0}^{T^{\prime \prime}} \int_{2} e^{2 \lambda t} u^{2} d x d t
$$

We can easily verify that $D_{x}^{x} \varphi(|\alpha| \leqq s)$ tends to $D_{x}^{x} u$ in $L^{2}(\mathscr{D})$ as $\varepsilon$ tends to zero. Applying Gårding's inequality for the second term in the left hand side of (3), we get

$$
\begin{aligned}
& \lim _{\varepsilon \rightarrow 0} \int_{0}^{\tau^{\prime \prime}} \int_{g} \sum_{|\alpha| \equiv \sum_{\alpha}}(-1)^{|\alpha|} a_{\alpha \beta} D D_{x}^{\beta} u_{x}^{\alpha} \varphi d x d t \\
& =\int_{0}^{T^{\prime \prime \prime}} \int_{\mathscr{2}} e^{2 \lambda t} \sum_{|\alpha| \equiv s}(-1)^{|x|} a_{\alpha \beta} D_{x}^{\beta} u D_{x}^{\alpha} u d x d t \\
& \geqq c_{1} \int_{0}^{T^{\prime \prime}} \int_{g} e^{2 \lambda t} \sum_{|\alpha| \leqq s}\left|D_{x}^{x} u\right|^{2} d x d t-c_{2} \int_{0}^{T^{\prime \prime}} \int_{Z^{2}} e^{2 \lambda t} u^{2} d x d t
\end{aligned}
$$

for some positive constants $c_{1}$ and $c_{2}$ depending only on $L$. Here we used that $s$ is even. Further, by using Aronson's argument [2], we have

$$
\begin{aligned}
& \lim _{\varepsilon \rightarrow 0}\left|\int_{2} u(x, T) \varphi(x, T) d x-\int_{2} u\left(x, T^{\prime \prime}\right) \varphi\left(x, T^{\prime \prime}\right) d x\right| \\
= & \frac{1}{2} \int_{2} u^{2}(x, 0) d x-\frac{1}{2} \int_{2} e^{2 \lambda I^{\prime \prime}} u^{2}\left(x, T^{\prime \prime}\right) d x .
\end{aligned}
$$

Finally for the last term of the right hand side of (3), it holds from (4) that

$$
\left|\lim _{\varepsilon \rightarrow 0} \int_{0}^{T^{\prime \prime}} \int_{g} f \varphi d x d t\right| \leqq \eta \frac{k}{2} \int_{0}^{T^{\prime \prime}} \int_{g} e^{2 \lambda t} \sum_{|\alpha| \leqq s}\left|D_{x}^{x} u\right|^{2} d x d t+\frac{1}{2 \eta} \int_{0}^{T^{\prime \prime \prime}} \int_{2} e^{2 \lambda t} u^{2} d x d t
$$

for any positive constant $\eta$. Hence, making $\varepsilon \rightarrow 0$ in (3), we obtain

$$
\begin{aligned}
& -\lambda \int_{0}^{T^{\prime \prime}} \int_{2} e^{2 \lambda t} u^{2} d x d t-c_{1} \int_{0}^{T^{\prime \prime}} \int_{2} e^{2 \lambda t} \sum_{\mid \alpha_{1} \sum_{3}}\left|D_{x}^{\alpha} u\right|^{2} d x d t+c_{2} \int_{0}^{T^{\prime \prime \prime}} \int_{2} e^{2 \lambda t} u^{2} d x d t \\
& \geqq \frac{1}{2} \int_{2} u^{2}(x, 0) d x-\frac{1}{2} \int_{2} e^{2 \lambda T^{\prime \prime}} u^{2}\left(x, T^{\prime \prime}\right) d x-\eta \frac{k}{2} \int_{0}^{T^{\prime \prime}} \int_{g} e^{2 \lambda t} \sum_{|\alpha| \equiv s}\left|D_{x}^{\alpha} u\right|^{2} d x d t \\
& -\frac{1}{2 \eta} \int_{0}^{\eta^{\prime \prime \prime}} \int_{2} e^{2 \lambda t} u^{2} d x d t
\end{aligned}
$$

for any positive $T^{\prime \prime}$. Therefore, the condition (5) implies that 


$$
\begin{gathered}
-\left(\lambda-c_{2}-\frac{1}{2 \eta}\right) \int_{0}^{\infty} \int_{\mathscr{g}} e^{2 \lambda t} u^{2} d x d t-\left(\left(c_{1}-\eta \frac{k}{2}\right) \int_{0}^{\infty} \int_{\mathscr{g}} e^{2 \lambda t} \sum_{|x| \leqq s}\left|D_{x}^{\alpha} u\right|^{3} d x d t\right. \\
\geqq \frac{1}{2} \int_{g} u^{2}(x, 0) d x,
\end{gathered}
$$

which gives the following for a sufficiently small $\eta(>0)$;

$$
-\left(\lambda-c_{2}-\frac{1}{2 \eta}\right) \int_{0}^{\infty} \int_{\mathscr{2}} e^{2 \lambda t} u^{2} d x d t \geqq \frac{1}{2} \int_{\mathscr{D}} u^{2}(x, 0) d x .
$$

Since $\lambda$ is arbitrary as far as positive, we make $\lambda \rightarrow+\infty$ and can see that

$$
\int_{0}^{\infty} \int_{\mathscr{D}}^{2 \lambda t} e^{2} d x d t=0
$$

which shows that $u$ vanishes throughout $\Omega$.

4. Remarks.

1. In Theorem, it is sufficient to assume (5) for a sufficiently large $\lambda$, namely for a $\lambda>c_{2}+\frac{k}{4 c_{1}}$.

2. In the previous paper [3] on a parabolic equation of the form

$$
\frac{\partial u}{\partial t}-\sum_{|\alpha| \leqq 2} a_{\alpha} D_{x}^{\alpha} u=f
$$

for an even $s$, we proved the asymptotic behavior similar to Theorem in this paper. Our Theorem is regarded as an improvement of a result in [3].

\section{REFERENCES}

[1] D. G. Aronson, On the Green's function for second order parabolic differential equations with discontinuous coefficients. Bulletin of the American Mathematical Society 69 (1963), pp. 841-847.

[2] D. G. Aronson, Uniqueness of positive weak solutions of second order parabolic equations. Annales Polonici Mathematici XVI (1965), pp. 285-303.

[3] L. S. Chen, Asymptotic behavior of solutions of parabolic equations of higher order. ( to appear in Pacific J. Math.).

[4] T. Kuroda, Note on the uniqueness property of weak solutions of parabolic equations. (to appear in Nagoya Math. J.).

Department of Mathematics,

Taiwan Provincial Cheng-Kung University, Tainan

and

Mathematical Institute, Nagoya University, Nagoya 